\title{
Single-spin azimuthal asymmetry in exclusive electroproduction of $\phi$ and $\omega$ vector mesons on transversely polarized protons
}

\author{
W. Augustyniak* \\ Sołtan Institute for Nuclear Studies \\ E-mail: witeka@fuw.edu.pl
}

\begin{abstract}
Hard exclusive electro-production of $\phi$ and $\omega$ vector mesons was studied with the HERMES spectrometer at the DESY laboratory by scattering $27.6 \mathrm{GeV}$ positron and electron beams off a transversely polarized hydrogen target. The single-spin azimuthal asymmetry with respect to the transverse proton polarization $\mathrm{P}_{T}$ was measured. Two asymmetry amplitudes, $\mathrm{A}_{U T}^{\sin \left(\phi+\phi_{S}\right)}$ and $\mathrm{A}_{U T}^{\sin \left(\phi-\phi_{S}\right)}$, are presented as a function of the negative squared four momentum $\mathrm{Q}^{2}$ of the virtual photon, and of the Mandelstain variable and Bjorken x. The experimental conditions as well as the influence of other processes on the results are discussed and the data are compared with theoretical model predictions based on Generalized Parton Distributions (GPDs) .
\end{abstract}

XVIII International Workshop on Deep-Inelastic Scattering and Related Subjects, DIS 2010

April 19-23, 2010

Firenze, Italy

\footnotetext{
${ }^{*}$ Speaker.
} 


\section{Introduction}

Lepton-induced reactions using a longitudinally and/or transversely polarized proton target provide important information about the nucleon spin structure. From inclusive data the structure functions $\mathrm{g}_{1}$ and $\mathrm{g}_{2}$ were determined. Semi-inclusive data yielded the important Collins and Sivers functions. Hard exclusive electroproduction of vector mesons provides information about GPDs, in particular about the GPD E which is a consequence of the fact that in an exclusive experiment with a transversely polarized target a target spin-flip is involved. In spite of the existence of certain common elements in semi-inclusive and exclusive analyses using a the transversely polarized target presented in the paper of M.Diehl and S.Sapeta [1], the theoretical descriptions of the two processes are different. In the case of the semi-inclusive process the cross section can be factorized into the hard-scattering process amplitude multiplied by parton densities and fragmentation functions which explicitly depend on the transverse parton momentum, while the exclusive meson production amplitude is a convolution of the hard scattering subprocess amplitude with GPDs and the lightcone distribution amplitude of the produced meson. Different hierarchies were predicted for the subprocess amplitudes in these two cases.

The goal of this analysis was the construction of the asymmetry amplitude $\mathrm{A}_{U T}$ that is closely related to the GPD E. The possibility of extracting from $\mathrm{A}_{U T}$ information on the quark total angular momenta, at least for $\mathrm{u}$ and $\mathrm{d}$ quarks, was first discussed by Ellinghaus et al [7]. $\mathrm{A}_{U T}$ is related to a convolution involving the GPDs $\mathrm{H}$ and $\mathrm{E}$. While $\mathrm{H}$ is well constrained by existing experimental data, $\mathrm{E}$ is mainly obtained from postulated asymmetries [3, 4] but not verified by experiment. $\mathrm{A}_{U T}$ for the $\rho^{0}$ meson was first measured by the Hermes Collaboration [5]. The target spin asymmetry depends on the azimuthal angles $\phi$ and $\phi_{S}$. In Fig. 1 both angles are defined: $\phi$ is the angle between the scattering and production planes and $\phi_{S}$ the angle between the scattering plane and the target spin vector.

In the experiment the target proton is polarized perpendicularly to the beam. Calculations are usually done in the frame, where the proton spin is perpendicular to the virtual photon direction. These two frames are rotated with respect to each other by an angle $\theta$ determined by the $x_{B j}$ and $Q^{2}$ values:

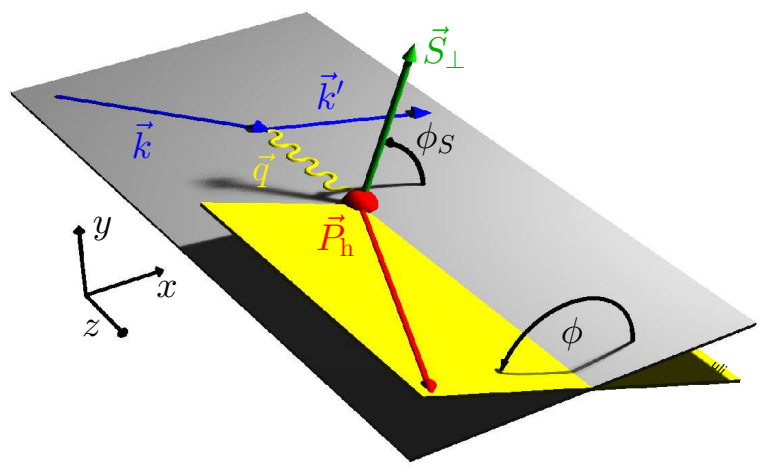

Figure 1: Definition of azimuthal angels $\phi$ and $\phi_{S}$ ( signs agree with Trento convention [2]). $\sin \theta=\gamma \sqrt{\frac{1-y-\frac{1}{4} y^{2} \gamma^{2}}{1+\gamma^{2}}}, \gamma=2 x_{B j} M_{p} / Q$, where $\mathrm{Q}^{2}$ is the negative square of the four-momentum of the virtual photon, $x_{B j}$ is the Bjorken scaling parameter and $\mathrm{y}=\left(\mathrm{E}^{\text {beam }}-\mathrm{E}^{\prime}\right) / \mathrm{E}^{\text {beam }}$, where $\mathrm{E}^{\prime}$ is the energy of the scattered positron. In the Hermes kinematic region the angle $\theta$ is small, of the order $\sim 0.01$.

This analysis uses formula (34) of ref. [1] as a starting point. In this formula the differential cross section contains terms independent of the transverse target polarization $P_{T}$ and terms dependent on it, the latter being the product of two functions: $\mathrm{f}\left(\phi, \phi_{S}\right) \mathrm{g}\left(\mathrm{Q}^{2}, \mathrm{x}_{B j}\right)$. The functions depending 
on $\phi$ and $\phi_{S}$ were ordered as $\sin \left(n \phi_{S} \pm m \phi\right)$ functions. The amplitudes of the particular sinusoidal terms are written as functions of the polarized photo-absorption cross sections or interference terms $\sigma_{m n}^{i j}$ and have a physical interpretation. The lower indices describe the helicities of the virtual photon and vector mesons, the upper ones the proton helicities. These amplitudes depend only on the kinematic variables $\mathrm{Q}^{2}, \mathrm{x}_{B j}$, y and the depolarization parameter $\varepsilon$. The terms depending on $P_{T}$ read:

$$
\begin{array}{r}
-\frac{P_{T}}{\sqrt{1-\sin ^{2}(\theta) \sin ^{2}\left(\phi_{S}\right)}}\left[\sin \phi_{S} \cos \theta \sqrt{\varepsilon(1+\varepsilon)} \operatorname{Im} \sigma_{+0}^{+-}\right. \\
+\sin \left(\phi-\phi_{S}\right)\left(\cos \theta \operatorname{Im}\left(\sigma_{++}^{+-}+\varepsilon \sigma_{00}^{+-}\right)+\frac{1}{2} \sin \theta \sqrt{\varepsilon(1+\varepsilon)} \operatorname{Im}\left(\sigma_{+0}^{++}-\sigma_{+0}^{--}\right)\right) \\
+\sin \left(\phi+\phi_{S}\right)\left(\cos \theta \frac{\varepsilon}{2} \operatorname{Im} \sigma_{+-}^{+-}+\frac{1}{2} \sin \theta \sqrt{\varepsilon(1+\varepsilon)} \operatorname{Im}\left(\sigma_{+0}^{++}-\sigma_{+0}^{--}\right)\right) \\
+\sin \left(2 \phi-\phi_{S}\right)\left(\cos \theta \sqrt{\varepsilon+(1+\varepsilon)} \operatorname{Im} \sigma_{+0}^{-+}+\frac{1}{2} \sin \theta \varepsilon \operatorname{Im} \sigma_{+-}^{++}\right) \\
\left.+\sin \left(2 \phi+\phi_{S}\right) \frac{1}{2} \sin \theta \varepsilon \operatorname{Im} \sigma_{+-}^{++}+\sin \left(3 \phi-\phi_{S}\right) \cos \theta \frac{\varepsilon}{2} \operatorname{Im} \sigma_{+-}^{-+}\right]
\end{array}
$$

The goal of our analysis was the determination of the amplitudes of the sinusoidal terms. The amplitude of the $\sin \left(\phi-\phi_{S}\right)$ modulation, called $\mathrm{A}_{U T}$, is particularly interesting from the theoretical point of view. It depends on the terms $\sigma_{++}^{+-}$and $\sigma_{00}^{+-}$which describe the transitions $\gamma_{T}^{*} \rightarrow V_{T}$ as well as $\gamma_{L}^{*} \rightarrow V_{L}$, with spin-flip of the proton. Here, we will focus on exclusive production of the vector mesons $\phi$ and $\omega$ in electroproduction with a transversely polarized proton target at HERMES.

\section{Experimental results}

The data were accumulated with the HERMES forward spectrometer during the running period 2002-2005. The $27.6 \mathrm{GeV}$ positron (electron) beam was scattered off a transversely polarized hydrogen target with an average polarization of 0.72 . Exclusive $\phi$ vector mesons were identified using the same cuts and procedures as in the analysis of similar measurements with an unpolarized target [6]. Events with positron (electron) and two oppositely charged kaons were selected. The separation of leptons and hadrons was better than 99\%. RICH information was used for kaon identification. Exclusive events were selected using the $\phi$ region for mass $\mathrm{M}_{K K}$ as well as $\Delta E=\frac{M_{x}^{2}-M_{p}^{2}}{2 M_{p}}<0.6 \mathrm{GeV}$. Two additional cuts were introduced. The fact that the kinetic energy $\left(M_{K K}-2 M_{K}\right)$ of the $\phi$ is small, is equivalent to a low opening angle of the two kaons in the laboratory frame. A cut on the opening angle hence reduces the non-resonant background contribution. The cut on the $\phi$ meson momentum $\left(\mathrm{P}_{\phi}>7.5 \mathrm{GeV}\right)$ reduces the SIDIS contribution. The kinematic region was chosen as $\mathrm{Q}^{2}>1 \mathrm{GeV}^{2}, \mathrm{t}^{\prime}<0.5 \mathrm{GeV}^{2}$ and $\mathrm{W}>5 \mathrm{GeV}$. The results obtained with the applied cuts are presented in Fig. 2. One can see from the fitted Breit-Wigner function for the invariant mass spectra that non-resonant contribution for exclusive spectrum is negligible. The fraction of SIDIS events as determined by PYTHIA simulations is $1.6 \%$. As the non-resonant and SIDIS contributions are negligible the errors of $\mathrm{A}_{U T}$ in the results presented in Fig. 2, for $\phi$ mesons come mainly from statistics. In Ref. [6], the spin density matrix elements were presented for $\phi$ mesons. From these data the ratio $\sigma_{L} / \sigma_{T}$ was determined. It was also stated that s-channel helicity in the case of $\phi$ meson is conserved, permitting also to separately determine the amplitudes 


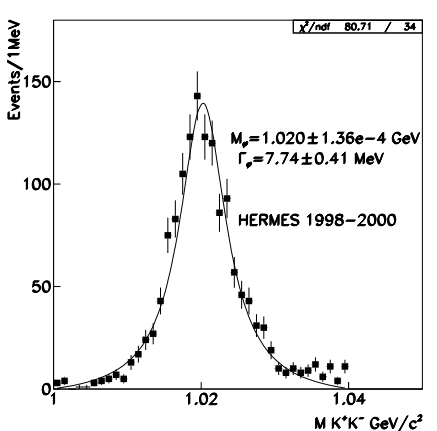

(a) $\mathrm{M}_{K K}(\mathrm{GeV})$

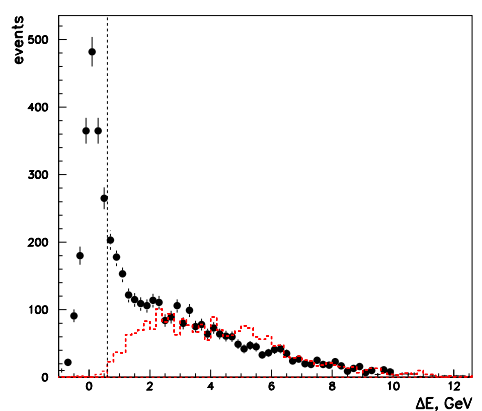

(b) $\Delta \mathrm{E}(\mathrm{GeV})$

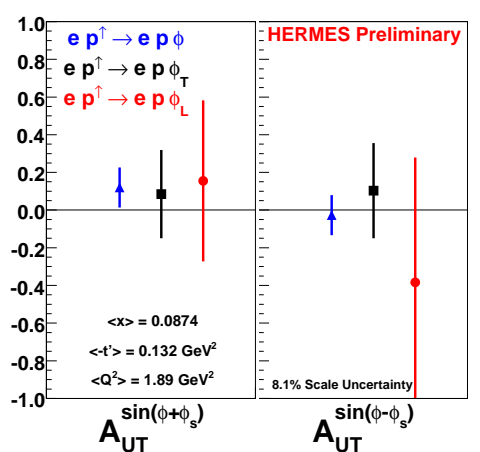

(c) $\mathrm{A}_{U T}$

Figure 2: (a) The two-kaon invariant mass for exclusive events after cuts described in the text; (b) $\Delta \mathrm{E}$ spectra and simulated SIDIS background (dashed line ) fitted to experiment ; (c) $\mathrm{A}_{U T}^{\sin \left(\phi \pm \phi_{S}\right)}$ amplitudes with separated transitions $\gamma_{L}^{*} \rightarrow \phi_{L}$ and $\gamma_{T}^{*} \rightarrow \phi_{T}$.
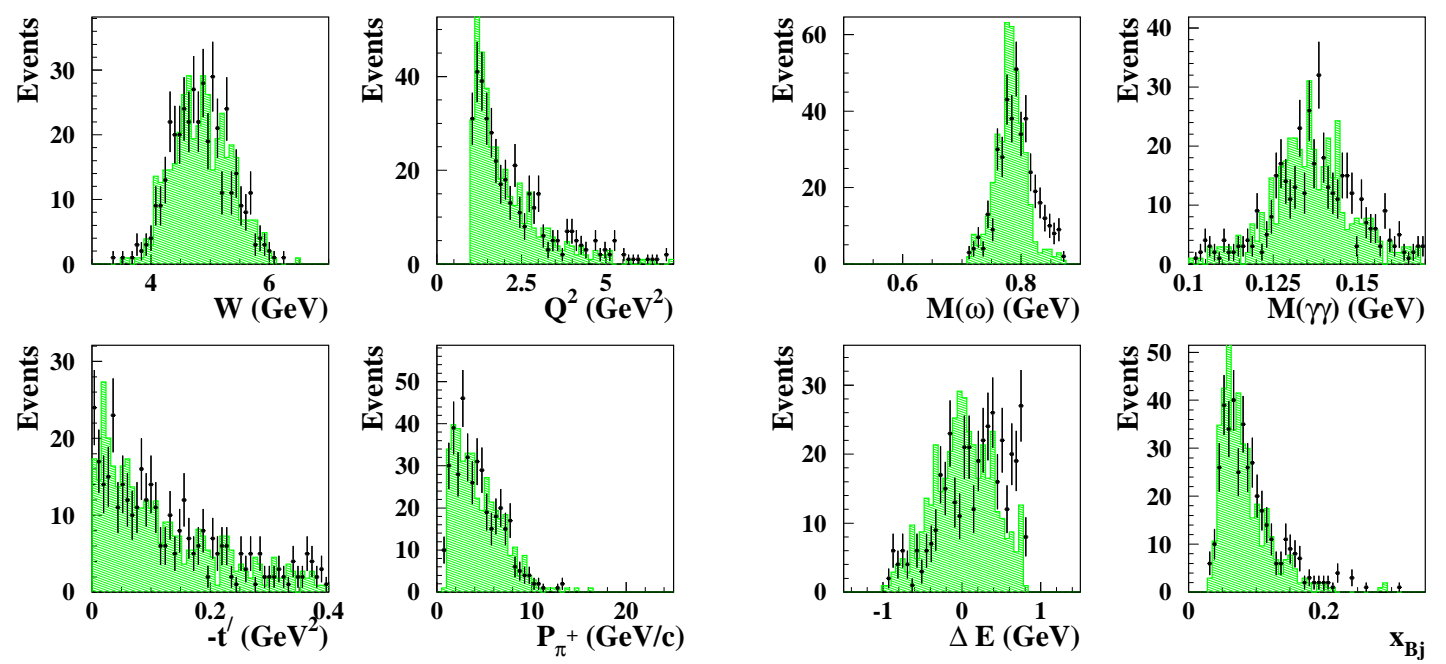

Figure 3: Kinematic distributions for exclusively produced $\omega$ vector mesons. Shaded (green) distributions show PYTHIA simulations. Experimental data points are shown by black circles.

$\mathrm{A}_{U T}^{\sin \left(\phi+\phi_{S}\right)}$ and $\mathrm{A}_{U T}^{\sin \left(\phi-\phi_{S}\right)}$ for the transitions $\gamma_{L}^{*} \rightarrow \phi_{L}$ and $\gamma_{T}^{*} \rightarrow \phi_{T}$, which are presented in Fig.2 as triangles together with amplitudes for the transition $\gamma_{T}^{*} \rightarrow \phi_{T}$ and $\gamma_{L}^{*} \rightarrow \phi_{L}$ shown as squares and circles, respectively. For the $\omega$ mesons, the decay $\omega \rightarrow \pi^{+}+\pi^{-}+\pi^{0}$ with $\pi^{0} \rightarrow \gamma+\gamma$ was used. The $\pi^{0}$ meson was reconstructed from two $\gamma$ clusters with invariant mass $0.11<M_{\gamma \gamma}<0.16 \mathrm{GeV}$. In this case information from the RICH detector was not used due to the fact that in the case of a three body decay the charged-pion momenta are mostly too low to allow $\pi^{ \pm}$identification. Also the calorimeter prefers higher momenta of $\pi^{0}$ [10]. For these reasons, the exclusive $\omega$ vector mesons were selected by cuts on the invariant mass spectrum $M\left(\pi^{+}, \pi^{-}, \pi^{0}\right)$ and the $\Delta \mathrm{E}$ spectrum. The kinematic distributions of the exclusively produced $\omega$ are presented in Fig. 3. Final results on the integrated values as well as on the $\mathrm{Q}^{2}, \mathrm{x}_{B j}$, and $\mathrm{t}^{\prime}$ dependences of various amplitudes are presented 
in Fig.4. Statistical and systematic errors are presented separately.

\section{Discussion of theoretical predictions}

The transverse-target-spin asymmetry for exclusively produced vector mesons, $\mathrm{A}_{U T}$, is also the subject of theoretical studies. Numerical results were predicted for $\phi$ and $\omega[8,9]$. The sign and value of $\mathrm{A}_{U T}$ depend on the type of produced meson. In the case of Ref. [9], the role of Next-to-Leading Order corrections was studied in the collinear approach. It was stated that in the case of $\omega$ these corrections are important. The calculations of Ref. [8] are performed close to the HERMES kinematic region. In these calculations the quark transverse degrees of freedom and Sudakov suppressions are taken into account. Including the quark transverse momenta provides a model-dependent regularization. Different contributions arise from the GPDs E for gluons, sea quarks and valence quarks. For $\phi$ mesons, it is assumed and argued that gluon and sea quark contributions have to cancel almost completely, should be very small or close to zero. One can see in Fig. 2 that the values of the measured asymmetry amplitudes $\mathrm{A}_{U T}, \mathrm{~A}_{U T}^{T}$ and $\mathrm{A}_{U T}^{L}$ confirm these assumptions, given the rather large uncertainties.

In the case of $\omega$ the predicted value is negative, $-0.12 \pm 0.05$ ( the uncertainty is given by those of the fit parameters which are constrained by data ), while the experimental value is $-0.22 \pm 0.16$. Due to the relatively large uncertainty of $\mathrm{A}_{U T}$ only one of the predictions of Ref. [8] can be eliminated.

\section{References}

[1] M. Diehl et al., Eur. Phys. J. C41 515 (2005).

[2] A. Bacchetta et al.,Phys. Rev. D70 117504 (2004).

[3] K. Goeke et al., Prog. Part. Nucl. Phys 47401 (2001).

[4] M. Diehl et al., Phys. Rep. 38841 (2003).

[5] A. Airapetian et al.,Phys. Lett. B679 100 (2009)

[6] W. Augustyniak et al., [hep-ex/0808.0669v4]

[7] F. Ellinghaus et al., Eur. Phys. J. C46 729 (2006)

[8] S.V. Goloskokov et al.,Eur.Phys.J. C59 800 (2008)

[9] M. Diehl et al., Eur. Phys.J. C52 933 (2007)

[10] A. Vandenbroucke

Ph.D.Thesis; DESY-THESIS-2007-003

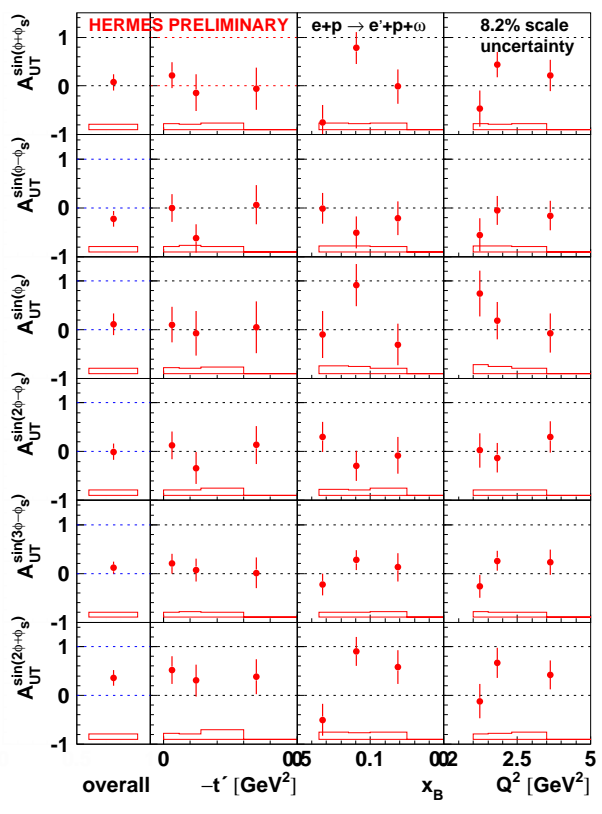

Figure 4: Results on integrated values as well as $\mathbf{Q}^{2}, \mathbf{x}_{B j}$, and $\mathbf{t}^{\prime}$ dependences of six asymmetry amplitudes determined for exclusive $\omega$ vector mesons . 